\title{
Una nueva especie de Cosmophyga Dognin (Lepidoptera, Geometridae) del norte de Chile
}

\author{
Héctor A. Vargas ${ }^{1}$ \\ ${ }^{1}$ Departamento de Recursos Ambientales, Facultad de Ciencias Agronómicas, Universidad de Tarapacá, Casilla 6-D, Arica, Chile; Becario MECE \\ Educación Superior, Ministerio de Educación, Gobierno de Chile; Programa de Pós-Graduação em Entomologia, Departamento de Zoologia, \\ Universidade Federal do Paraná, Curitiba-PR, Brasil; havargas@uta.cl
}

\begin{abstract}
A new species of Cosmophyga Dognin (Lepidoptera, Geometridae) from northern Chile. Cosmophyga cortesi sp. nov. is described from Azapa Valley, Arica Province, northern Chile, based on male and female adults. This is the first mention of Cosmophyga from Chile.

KEYWORDS. Ennominae; Neotropical; Taxonomy.

RESUMEN. Una nueva especie de Cosmophyga Dognin (Lepidoptera, Geometridae) del norte de Chile. Se describe Cosmophyga cortesi sp. nov. del Valle de Azapa, Provincia de Arica, norte de Chile, sobre la base de adultos macho y hembra. Este es el primer reporte de Cosmophyga para Chile.
\end{abstract}

PALABRAS CLAVE. Ennominae; Neotropical; Taxonomy.

Cosmophyga Dognin, 1912 es un pequeño género Neotropical de Ennominae (Geometridae) con cinco especies incluidas, las cuales están representadas en Colombia, Venezuela, Ecuador, Perú y Bolivia (Pitkin 2002). No ha sido reportado previamente para el territorio chileno. Sin embargo, algunos ejemplares de una especie no descrita han sido recientemente colectados en el Valle de Azapa, Provincia de Arica, norte de Chile. Entonces, este trabajo tiene por finalidad presentar una descripción de esta nueva especie.

\section{MATERIALY MÉTODO}

Los ejemplares examinados en este estudio fueron capturados en el valle de Azapa, Provincia de Arica, norte de Chile, entre los años 2003 y 2008, y han sido depositados en el Museo Nacional de Historia Natural de Santiago, Chile (MNNC), Museo de Zoología de la Universidad de Concepción (UCCC) y en la Colección Entomológica de la Universidad de Tarapacá (IDEA).

\section{RESULTADOS}

\section{Cosmophyga cortesi sp. nov.}

(Figs. 1, 2, 3, 4, 5)

Material tipo. Holotipo macho, CHILE, Arica: Azapa, Arica, Chile, Enero 2008, H.A. Vargas coll. (MNNC). Paratipos, CHILE, Arica: 1 hembra Azapa, Arica, Chile, diciembre 2003, H.A. Vargas coll. (MNNC); 1 macho Azapa, Arica, Chile, Diciembre 2005, H.A. Vargas coll.; 1 hembra Azapa, Arica, Chile, noviembre 2005, H.A. Vargas coll. (UCCC); 1 macho Azapa, Arica, Chile, enero 2007, H.A. Vargas coll., 1 macho Azapa, Arica, Chile, noviembre 2006, H.A. Vargas coll. (IDEA).
Diagnosis. Superficie dorsal de las alas parda grisácea; ala anterior con líneas antemedial y posmedial gris oscuras débilmente diferenciadas; macho carece de pincel de pelos en el esternito III; genitalia del macho con la yuxta no proyectada distalmente entre los procesos del anellus, costa con una proyección distal espiniforme esclerotinizada.

Macho. (Fig. 1) Cabeza con ojos compuestos gris oscuro; frente parda grisácea, con algunas escamas gris oscuro; vértex pardo grisáceo, ápice de las escamas dirigido hacia abajo; occipucio pardo grisáceo, ápice de las escamas dirigido hacia adelante; quetosemas en dos pequeños grupos, cada uno posterior a la base de la antena, cerca del margen del ojo compuesto; antena filiforme, escapo, pedicelo y dorso del flagelo pardo blanquecino, superficie ventral del flagelo desprovista de escamas; palpo labial pequeño, pardo grisáceo. Protórax y mesotórax pardo grisáceo, metatórax pardo blanquecino; escamas de las patagias estrechas, elongadas y dirigidas hacia arriba; escamas de las tégulas estrechas, elongadas y dirigidas hacia atrás; escamas del metatórax aplanadas y pequeñas. Ala anterior: subtriangular, margen externo débilmente proyectado hacia afuera a la altura de M3; superficie dorsal parda grisácea, línea antemedial y posmedial gris oscura débilmente diferenciada; mancha discal parda oscura, pequeña, débilmente diferenciada; superficie ventral pardo blanquecina, con algunas escamas pardo oscuras dispersas, principalmente concentradas distalmente. Ala posterior: subtriangular, margen externo débilmente proyectado hacia afuera a la altura de M3; coloración de la superficie dorsal similar al ala anterior, pero carece de línea antemedial y presenta escamas pardo blanquecinas cerca del margen anterior; 


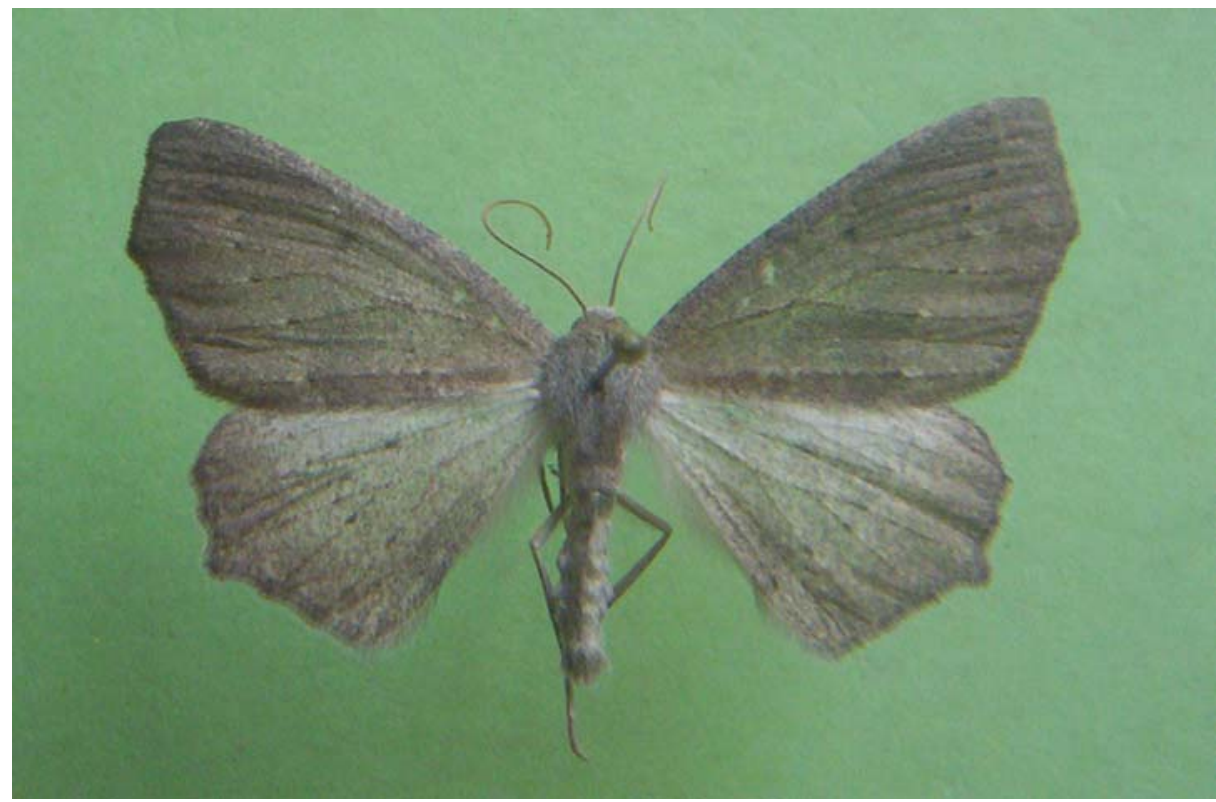

Fig. 1. Cosmophyga cortesi sp. nov. Holotipo macho en vista dorsal.

superficie ventral pardo blanquecina con escamas pardo oscuras dispersas. Patas pardo blanquecinas, con algunas escamas pardo grisáceas salpicadas; tibia protorácica con epífisis; tibia mesotorácica con un par de espinas; tibia metatorácica con dos pares de espinas. Abdomen pardo

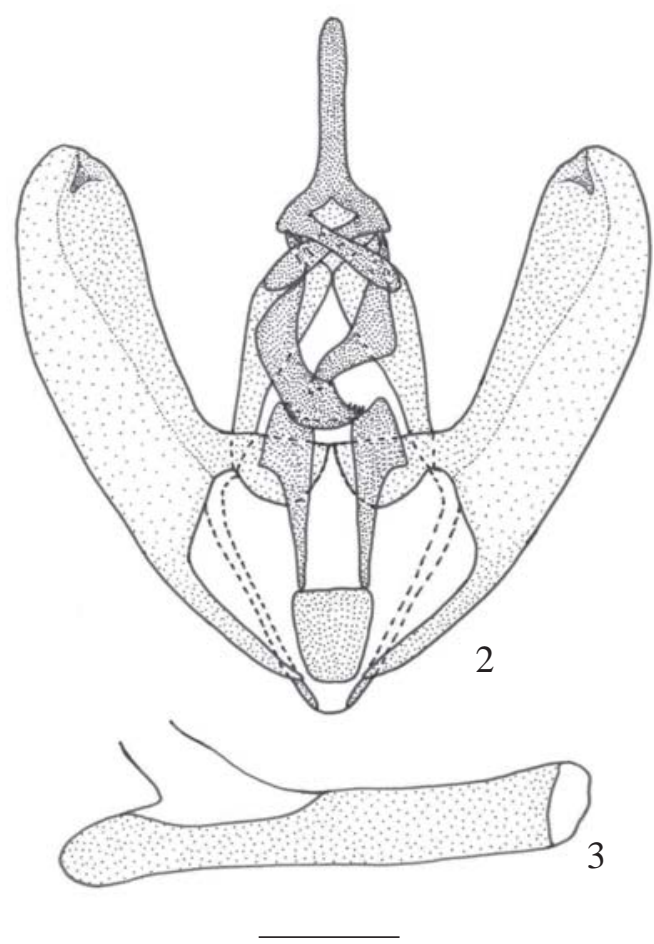

Figs. 2-3. Genitalia del macho de C. cortesi $\mathbf{s p . ~ n o v . ; ~ 2 ) ~ v i s t a ~ v e n t r a l , ~}$ aedeagus removido; 3) aedeagus en vista lateral; escala: $0,5 \mathrm{~mm}$. blanquecino con escamas pardo grisáceas dispersas, hilera de escamas piliformes en esternito III ausente.

Genitalia del macho. (Figs. 2, 3) Uncus elongado, aplanado, ápice redondeado; socii bien diferenciados, digitiformes, esclerotinizados, cerca de la mitad la longitud del uncus; gnathos en forma de $\mathrm{V}$, ápice ventral débilmente curvado y dentado distalmente; tegumen estrecho, hendido anteriormente, claramente separado del saccus; brazos laterales del saccus estrechos, bien diferenciados, ventralmente membranoso; yuxta rectangular; anellus con un par de procesos esclerotinizados, estrechos en la base, dilatados distalmente; valva estrecha, margen distal redondeado; costa amplia, más esclerotinizada que lo restante de la valva, con una proyección distal espiniforme esclerotinizada; transtilla semicircular. Aedeagus cilíndrico, longitud cerca de 1,5 veces la costa; vesica sin cornuti.

Hembra. Similar al macho.

Genitalia de la hembra. (Fig. 4, 5) Antrum corto, aplanado; ductus bursae membranoso, cilíndrico; corpus bursae membranoso, subesférico; signum de base elipsoidal, inserto ventralmente en la base del corpus bursae, margen distal dentado, proyectado internamente en la cavidad del corpus bursae; ductus seminalis inserto en la base del ductus bursae; lamella postvaginalis circular, aplanada, margen anterior proyectado hacia adelanate; apófisis anteriores elongadas, cerca de 2/3 la longitud del ductus bursae; apófisis posteriores elongadas, longitud similar al ductus bursae; papilas anales pilosas, débilmente esclerotinizadas.

Distribución. Conocida sólo de la localidad tipo: Azapa (18 34'S; $\left.70^{\circ} 00^{\prime} \mathrm{W}\right)$, Arica, norte de Chile.

Etimología. El nombre de esta especie ha sido dedicado a la memoria del brillante entomólogo chileno, profesor Raúl Cortés Peña. 


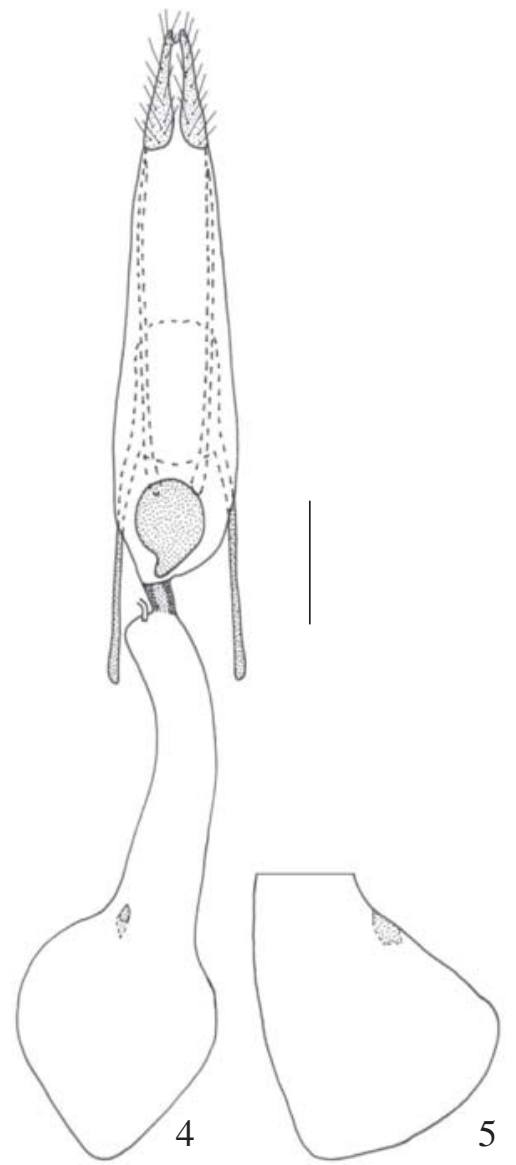

Figs. 4-5. Genitalia de la hembra de $C$. cortesi $\mathbf{s p . ~ n o v . ; ~ 4 ) ~ v i s t a ~ v e n t r a l ; ~}$ 5) corpus bursae en vista lateral; escala: $1 \mathrm{~mm}$.

\section{DISCUSIÓN}

Cosmophyga cortesi corresponde a la primera especie de este género descrita de Chile. La maculación alar de C. cortesi difiere ligeramente de lo mencionado para las restantes especies de Cosmophyga por Pitkin (2002), quien señala que las venas son más oscuras que el resto del ala, rasgo no evidente en $C$. cortesi. Además, $C$. cortesi es hasta ahora la única especie descrita de Cosmophyga que carece de pincel de pelos en el esternito III del macho. Pese a estas peculiaridades, la morfología genital del macho de la nueva especie aquí descrita permite razonablemente incluirla en este género. La genitalia del macho de $C$. cortesi es notablemente similar a la de Cosmophyga monastica (Dognin, 1893). Sin embargo, la yuxta se presenta proyectada distalmente entre los procesos del anellus en $C$. monastica, no así en $C$. cortesi. Además, la proyección distal de la costa es de mayor tamaño en $C$. cortesi.

Agradecimientos. Al Dr. Axel Hausmann, Zoologische Staatssammlung Muenchen, Alemania, y al Dr. Luis E. Parra, Universidad de Concepción, Chile, por el constante apoyo brindado al autor durante el estudio de la taxonomía de Geometridae en el norte de Chile; a los revisores de este manuscrito, por sus amables comentarios.

\section{REFERENCIA}

Pitkin, L. M. 2002. Neotropical Ennominae moths: a review of the genera (Lepidoptera: Geometridae). Zoological Journal of the Linnean Society 135: 121-401. 